\title{
Results of Channel Error Profiles for DECT
}

\author{
Pedro M. Crespo, R. Mann Pelz, J. P. Cosmas, and J. García-Frías
}

\begin{abstract}
This letter presents the main statistical characterization of the underlying error process obtained in the case of the Digital European Cordless Telecommunications (DECT) radio system. By simulation of the transmission link, error sequences are generated for different channel parameters. Relevant statistics are then computed for the purpose of efficient channel coding design and evaluation.
\end{abstract}

\section{INTRODUCTION}

$\mathbf{T}$ HE Digital European Cordless Telecommunications (DECT) system will provide a framework for high density, cordless, short range telecommunication links, covering a broad range of applications and environments either indoors or outdoors. As a consequence of the impairments introduced by the propagation conditions of a mobile radio channel, the use of error control techniques will be required as the means to improve the degradation suffered by the transmitted signal. Toward this end, the behavior of the DECT transmission system was first determined by a point-to-point DECT radio link simulation. From the generated error profiles, the most significant error statistics were then analyzed. The results obtained in this study should be seen as a basis for the design and evaluation of efficient channel coding schemes with the goal of achieving good service quality, when low bit rate digital video $(<32 \mathrm{~kb} / \mathrm{s})$ and the associated audio are transmitted through the DECT channel.

In the next section, the simulation of the radio link and the associated error process are described. In Section III, the relevant statistics are computed from the error process using a descriptive approach.

\section{LINK MODEL AND THE ERROR PROCESS}

The DECT time-division multiple access (TDMA) frame [1] has a duration of $10 \mathrm{~ms}$ and consists of $1152 \mathrm{~b}$ (bit rate of $1.152 \mathrm{Mb} / \mathrm{s}$ ) in which time slots of $420+60 \mathrm{~b}$ (guardtime of $60 \mathrm{~b}$ ) are assigned to 12 full-duplex logical channels. Only $320 \mathrm{~b}$ out of the 420 are used for actual transmission, leaving the remaining bits for synchronization, signalization, etc. This gives an information rate per logical channel of $32 \mathrm{~kb} / \mathrm{s}$. In the simulation, however, 420 bit slots per user have been considered. Notice that the $10 \mathrm{~ms}$ time separation between slots assigned to a user provides an additional time diversity protection that should be taken into account in the link simulation.

Paper approved by A. Duel-Hallen, the Editor for Communications of the IEEE Communications Society. Manuscript received March 20, 1993; revised March 23, 1994 and June 8, 1995.

The authors are with the Telefonica I+D, c/Emilio Vargas 6, 28043 Madrid, Spain.

Publisher Item Identifier S 0090-6778(96)05517-1.
The simulated DECT modulator consists of a Gaussian minimum shift keying (GMSK) modulator with a Gaussian $B T$ product of $0.5(1 / T=1.152 \mathrm{Mb} / \mathrm{s})$. The receiver comprises a fourth order Butterworth intermediate frequency (IF) filter with a normalized 3-dB bandwidth of $B_{I F} T=1$, a one-symbol differential demodulator, and a memoryless threshold detector. An oversampling rate of eight has been used throughout the time simulation. The radio channel is simulated as a finite transversal filter (TF) [2] with time delays close enough to verify the sampling theorem and with tap weight statistics determined by the scattering function $S(\tau, \nu)$ of the underlying channel. It is assumed that the scattering function equals the product of the delay power spectrum $Q(\tau)$ and the doppler power density spectrum $P(\nu)$. Additionally, a zero mean in the tap weight random processes has been assumed which is tantamount of not having a dominant line of sight (LOS) component (worst case situation). A classical $P(\nu)$ has been modeled

$$
P(\nu)= \begin{cases}\frac{1}{\pi \sqrt{f_{D_{\max }}^{2}-\nu^{2}}}, & \left|\frac{\nu}{f_{D_{\max }}}\right|<1 \\ 0, & \text { otherwise. }\end{cases}
$$

The link simulation has been carried out for four different channel parameters: two delay spread $t_{m}$ of values $0 \mathrm{~ns}$ and $100 \mathrm{~ns}$, and two maximum Doppler frequencies $f_{D_{\max }}$ of 2.5 $\mathrm{Hz}$ (speed: $1.5 \mathrm{~km} / \mathrm{h}$ ) and $25 \mathrm{~Hz}$ (speed: $15 \mathrm{~km} / \mathrm{h}$ ).

For the case of a delay spread of $100 \mathrm{~ns}$, the channel impulse response is approximated by a six-tap TF with time delays of $0.1 \mu \mathrm{s}$. The values of the corresponding power delay profile $Q\left(\tau_{i}\right)$ are given (in $\mathrm{dB}$ ) by $0,-4,-8,-11.5,-15.5$, and -19.5 (one-sided single exponential [3]).

It is important to mention at this point that we have imposed the constraint that the user only has access to a 32 $\mathrm{kb} / \mathrm{s}$ unprotected binary channel and therefore does not have access to the soft decisions at the output of the demodulator. In other words, we consider the whole DECT link as a binary (hard decisions) discrete-time channel with memory. We understand, however, that with soft decisions decoding, significant improvements can be achieved.

Therefore, the radio link will be completely described by the error process $\left\{e_{k}, k \in Z\right\}$. The error sequence $e$ represents a realization of the error process that can be related to the channel input and output as follows:

$$
\begin{aligned}
& \begin{array}{l}
e=\left\{\cdots, e_{1}, e_{0}, e_{1}, \cdots\right\} \\
\quad=\left\{\cdots, a_{-\eta-1} \oplus \hat{a}_{-\eta-1}, a_{-\eta} \oplus \hat{a}_{-\eta}, a_{-\eta+1} \oplus \hat{a}_{-\eta+1}, \cdots\right\}
\end{array} \\
& \text { where } \boldsymbol{a}=\left\{\cdots, a_{-1}, a_{0}, a_{1}, \cdots\right\} \text { and } \hat{\boldsymbol{a}}=\left\{\cdots, \hat{a}_{-1},\right. \\
& \left.\hat{a}_{0}, \hat{a}_{1}, \cdots\right\} \text { are the transmitted and received data sequences, } \\
& \text { respectively, and } \eta \geq 0 \text { is an appropriate delay. } \oplus \text { denotes }
\end{aligned}
$$




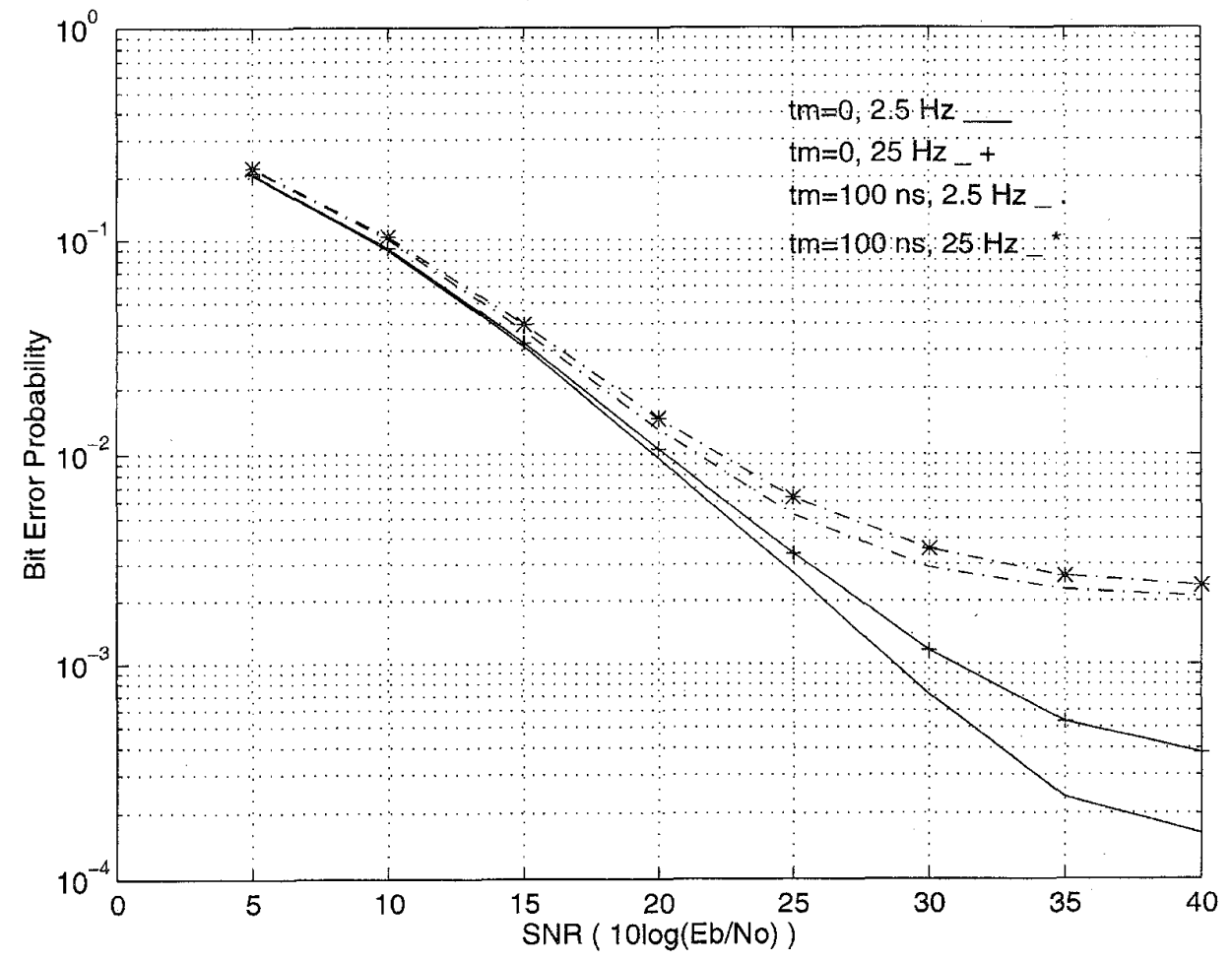

Fig. 1. BER versus SNR.

modulo 2 addition. An error is said to occur whenever $e_{k}=1$. The bit-error rate (BER) is simply given by

$$
p=\lim _{K \rightarrow \infty} \frac{1}{2 K+1} \sum_{j=-K}^{K} e_{j} .
$$

Fig. 1 plots the BER as a function of the signal-to-noise ratio (SNR). A $K=2.1 \times 10^{6}$ have been used throughout the analysis. Also, the simulation results that follow have been based on error profiles with a BER of approximately $10^{-3}$ $(\mathrm{SNR} \approx 30 \mathrm{~dB})$.

Since the probability of error $p$ is much less than 0.5 , more zeros are to be expected than ones in a typical error sequence. This leads to the notion of an error gap [4] as a means of describing the error process. A gap is defined as the string of $r-1$ consecutive zero's between two one's and having a length equal to $r$. Therefore, the binary error process can be likewise described in terms of the corresponding successive gap lengths $g_{i}$.

In general, a stationary gap process can be adequately described by the multigap-length mass distribution, $f^{s}(r)$ [5] defined as the probability of occurrence of $s$ consecutive gaps with a length $r$ equal to the sum of the composite gap lengths $(r \geq s)$. Notice that with the above notation, the probability of occurrence of a single gap of length $r \geq 1$ is denoted by $f(r)$. We also denote by $F(r)=\sum_{j=r+1}^{\infty} f(j)$ the error gap distribution (EGD). Fig. 2 shows the complementary EGD, i.e., $1-F(r)=\sum_{j=1}^{r} f(j)$ which gives the probability of having a gap of length less than or equal to $r$.

For those cases where successive gap lengths are uncorrelated, i.e., the gap process is renewal, the description of the gap process is further reduced and is completely characterized by $f(r)$. Fig. 3 plots the covariance of successive gap lengths $\operatorname{cov}\left(g_{k} g_{k+n}\right)$ for the 100 ns power delay spread channel (the same behavior has been observed for $t_{m}=0 \mathrm{~ns}$ ). Notice that in the case of a Doppler frequency of $2.5 \mathrm{~Hz}$, the covariance almost vanishes for $n \geq 1$, indicating that error process is quite close to a renewal process.

In the next section, the relevant statistics are computed as a function of the gap length distributions.

\section{ReleVANT Statistics AND Results}

The most frequently used measure to indicate the reliability of a block-coded forward error correction (FEC) system is the probability of block error. The channel statistic necessary to evaluate this in the case of random error correction is $P(m, n)$, the probability of exactly $m$ errors occurring in a block of $n$ digits. In the case of burst error correction, the statistic is $Q(l, n)$, the probability of an error burst of length $l$ occurring in a block of $n$ digits, where a frequently used definition of a burst in a block, is the length starting from the first error to the last error irrespective of the structure in between. The bit-error auto-correlation function is another relevant statistic that is useful in the design of bit interleaving. An additional insight into the error process can be gained by analyzing the burst error length distribution $C(l, d)$, which is defined as the probability of occurrence of a burst of length $l$. Here, we have adopted the burst definition given in [6]. A burst is specified by two quantities, the error density $0<d \leq 1$ and the burst length $l$. With the specified error density $d$ chosen, a burst is a sequence of binary digits, that starts and ends with an 


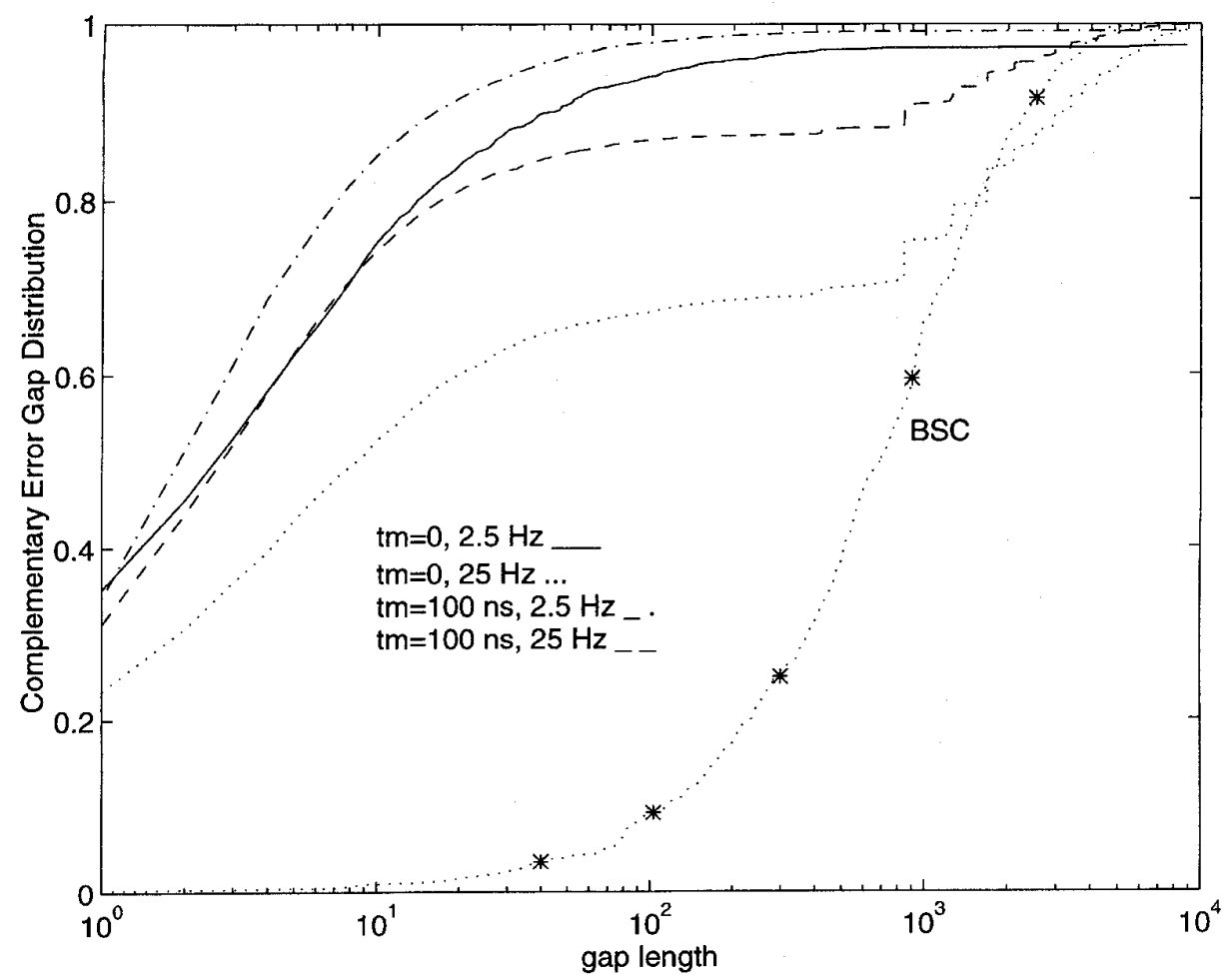

Fig. 2. Complementary EGD versus gap length for a $\mathrm{SNR} \approx 30 \mathrm{~dB}$.

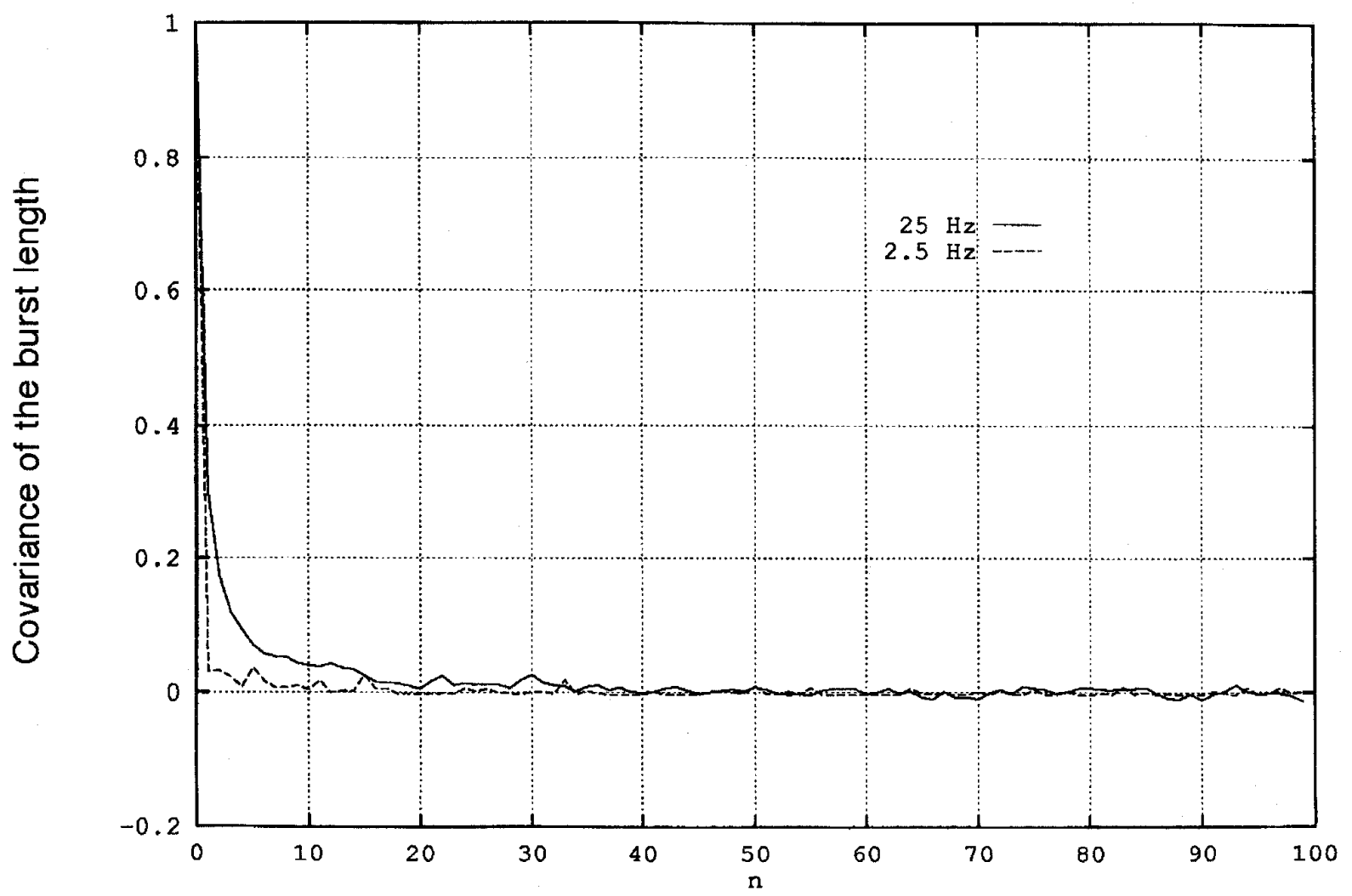

Fig. 3. $\operatorname{Cov}\left(g_{0}, g_{n}\right)$ versus $n$ for a delay spread $t_{m}=100$ ns and a SNR $\approx 30 \mathrm{~dB}$.

error, in which the ratio of the number of errors to the total number of digits of the sequence exceeds $d$. If the successive inclusions of the next error keeps the density above $d$, the burst is continued; the burst ends if further inclusions of an 
TABLE I

POlynomial COEPFICIENTS, In DEsCENDING POWERS OF $m$ FOR THE LS APPROXIMATION OF: $\log _{10}\left\{\sum_{j=m+1}^{n} P(j, n)\right\}$

\begin{tabular}{c|c|c|c||c|c|c}
\hline \hline$\alpha_{3}$ & $\alpha_{2}$ & $\alpha_{1}$ & $\alpha_{0}$ & $n$ & $f_{D_{\max }}$ & $t_{m}$ \\
\hline$-7.437 \mathrm{e}-03$ & $6.801 \mathrm{e}-02$ & $-5.192 \mathrm{e}-01$ & $-2.149 \mathrm{e}+00$ & 10 & $25 \mathrm{~Hz}$ & 0 ns \\
\hline$-9.814 \mathrm{e}-04$ & $1.820 \mathrm{e}-02$ & $-3.450 \mathrm{e}-01$ & $-2.077 \mathrm{e}+00$ & 15 & $25 \mathrm{~Hz}$ & 0 ns \\
\hline$-1.042 \mathrm{e}-03$ & $2.273 \mathrm{e}-02$ & $-2.753 \mathrm{e}-01$ & $-1.9293 \mathrm{e}+00$ & 30 & $25 \mathrm{~Hz}$ & 0 ns \\
\hline $4.339 \mathrm{e}-03$ & $-5.683 \mathrm{e}-02$ & $-1.244 \mathrm{e}-01$ & $-2.515 \mathrm{e}+00$ & 10 & $2.5 \mathrm{~Hz}$ & 0ns \\
\hline$-1.367 \mathrm{e}-03$ & $1.174 \mathrm{e}-02$ & $-2.227 \mathrm{e}-01$ & $-2.395 \mathrm{e}+00$ & 15 & $2.5 \mathrm{~Hz}$ & $0 \mathrm{~ns}$ \\
\hline$-8.343 \mathrm{e}-04$ & $1.7107 \mathrm{e}-02$ & $-1.985 \mathrm{e}-01$ & $-2.243 \mathrm{e}+00$ & 30 & $2.5 \mathrm{~Hz}$ & $0 \mathrm{~ns}$ \\
\hline$-2.301 \mathrm{e}-03$ & $2.955 \mathrm{e}-03$ & $-2.319 \mathrm{e}-01$ & $-1.808 \mathrm{e}+00$ & 10 & $25 \mathrm{~Hz}$ & $100 \mathrm{~ns}$ \\
\hline$-2.388 \mathrm{e}-03$ & $2.405 \mathrm{e}-02$ & $-2.449 \mathrm{e}-01$ & $-1.707 \mathrm{e}+00$ & 15 & $25 \mathrm{~Hz}$ & $100 \mathrm{~ns}$ \\
\hline$-7.881 \mathrm{e}-04$ & $1.673 \mathrm{e}-02$ & $-1.910 \mathrm{e}-01$ & $-1.575 \mathrm{e}+00$ & 30 & $25 \mathrm{~Hz}$ & $100 \mathrm{~ns}$ \\
\hline$-7.349 \mathrm{e}-03$ & $3.716 \mathrm{e}-02$ & $-2.400 \mathrm{e}-01$ & $-1.941 \mathrm{e}+00$ & 10 & $2.5 \mathrm{~Hz}$ & $100 \mathrm{~ns}$ \\
\hline$-1.974 \mathrm{e}-03$ & $9.505 \mathrm{e}-03$ & $-1.378 \mathrm{e}-01$ & $-1.902 \mathrm{e}+00$ & 15 & $2.5 \mathrm{~Hz}$ & $100 \mathrm{~ns}$ \\
\hline$-7.837 \mathrm{e}-04$ & $1.406 \mathrm{e}-02$ & $-1.411 \mathrm{e}-01$ & $-1.773 \mathrm{e}+00$ & 30 & $2.5 \mathrm{~Hz}$ & $100 \mathrm{~ns}$ \\
\hline
\end{tabular}

error reduces the error density below $d$. Finally, the burst does not begin with an error belonging to the previous burst.

The computation of the above relevant statistics can be obtained by inference from the error sequences. However, this will be a cumbersome operation (except for the bit correlation), since it would require almost a bit-by-bit analysis of the error sequence. It can be shown [4] that $P(m, n)$ can be expressed as a function of multigap distributions in the following way:

$$
P(m, n)=p \sum_{j=m-1}^{n-1}(n-j)\left(f^{m-1}(j)-2 f^{m}(j)+f^{m+1}(j)\right)
$$

$1 \leq m \leq n$, with the convention $f^{0}(j)=\delta(j)$.

The computer simulation shows that the log of the block error probability, in the case of having a block code capable of correcting up to $m$ random errors in a block of length $n$, can be approximated by a third degree polynomial in $m$ (least squares (LS) fitting)

$$
\log _{10}\left\{\sum_{j=m+1}^{n} P(j, n)\right\}=\alpha_{3} m^{3}+\alpha_{2} m^{2}+\alpha_{1} m+\alpha_{0}
$$

where $m \leq n$. The values of the $\left\{\alpha_{i}\right\}$ 's are given in Table I.

The computation of $Q(m, n)$ and $C(l, d)$ is much more cumbersome than in the case of $P(m, n)$ since it involves, beside multigap distributions, joint and conditional gap distributions [4]. However, in cases of low Doppler frequencies $\left(f_{D_{\max }} \leq 2.5 \mathrm{~Hz}\right)$, one can infer from the curves of Fig. 2 that a renewal assumption on the gap process is valid. This assumption substantially simplifies the computation of the above two quantities since then they can be expressed only in terms of $f(\nu)$. It can be shown that

$$
Q(l, n)=p \sum_{j=2}^{l} \sum_{i=0}^{n-l}(1-F(i+1)) V(j, l)(1-F(n-l-i))
$$

where $V(j, l)$ can be computed by

$$
V(j, l)=\sum_{i=1}^{l-j+1} f(i) V(j-1, l-i), 2 \leq j \leq l
$$

and with $V(2, l)=f(l-1)$ for $l \geq 2$.
TABLE II

Polynomial Coefficients, in Descending Powers of $l$ FOR THE LS APPROXIMATION OF: $\log _{10}\left\{\sum_{j=l+1}^{n} Q(j, n)\right\}$

\begin{tabular}{c|c|c||c|c|c}
\hline$\alpha_{2}$ & $\alpha_{1}$ & $\alpha_{0}$ & $d$ & $f_{D_{\max }}$ & $t_{m}$ \\
\hline \hline 0 & -0.4542 & 0 & 1 & $2.5 \mathrm{~Hz}$ & $0 \mathrm{~ns}$ \\
\hline 0 & -0.4664 & 0 & 1 & $2.5 \mathrm{~Hz}$ & $100 \mathrm{~ns}$ \\
\hline $5.065 \mathrm{e}-04$ & $-1.239 \mathrm{e}-01$ & $-2.131 \mathrm{e}-01$ & 0.5 & $2.5 \mathrm{~Hz}$ & $0 \mathrm{~ns}$ \\
\hline $2.204 \mathrm{e}-04$ & $-8.290 \mathrm{e}-02$ & $-2.107 \mathrm{e}-01$ & 0.5 & $2.5 \mathrm{~Hz}$ & $100 \mathrm{~ns}$ \\
\hline
\end{tabular}

Simulation results show that the $\log$ of the block error probability in the case of using a block code capable of correcting a single burst of length $l(l<n)$ in a block of length $n$, i.e., $\log _{10}\left\{\sum_{j=l+1}^{n} Q(j, n)\right\}$ can be approximated by a third order polynomial in $l$. Table II shows its coefficients for the different cases considered. Notice that only a $2.5 \mathrm{~Hz}$ Doppler frequency has been considered to comply with the renewal property of the underlying gap process. In addition, since it was observed from the results of computing $P(m, n)$ using a nonrenewal (3) and a renewal formulation [see [4] for the renewal expression of $P(m, n)$, that both results were quite close as long as $n$ was sufficiently small ( $n \leq 15$ ), only results for block lengths $n$ less than or equal to 15 are shown in Table II.

Similarly, $C(l, d)$ can be evaluated [6] as

$$
C(l, d)=\sum_{m=\max (\lfloor l d\rfloor, 2)}^{l} U(l, m)\left(1-F\left\lceil\frac{m+1}{d}-l\right\rceil\right)
$$

where $U(l, m)$ is the probability of a sequence of $l$ symbols with $m$ errors, which can be computed recursively according to

$$
U(l, m)=\sum_{j=m-1}^{\min (\lceil(m-1) / d\rceil, l-1)} U(j, m-1) U(l-j+1,2)
$$

and

$$
U(j, 2)=f(j-1)
$$

for $j=2,3, \cdots(l-\max (\lfloor l d\rfloor, 2)+2), m=$ $\max (\lfloor l d\rfloor, 2), \cdots, l-1, l$ and $l \geq 3$. The notations $\lfloor x\rfloor$ and $(\lceil x\rceil)$ denote the largest (smallest) integer less (greater) than $x$.

For the case of $d=1$, i.e., for error bursts consisting of sequences of consecutive one's, the $\log _{10}\left\{\sum_{j=l+1}^{\infty} C(j, d)\right\}$ which gives the occurrence probability of a burst of length greater than $l$, can be approximated by $\alpha_{1} l$, whereas for the other error density considered in the simulation $d=$ 0.5 , this quantity is better approximated by a second order polynomial. Table III gives the different coefficients for the different channel parameters. In the above LS approximations, the order of the polynomials has been chosen such that the normalized LS approximation error is always less than -30 $\mathrm{dB}$. Also, the approximations are valid for probabilities of error up to $10^{-6}$.

Finally, the bit correlation functions are given in Fig. 4. Two conclusions can be drawn from these curves. First, for large Doppler frequencies the memory of the channel is limited by the slot length of the DECT frame $(420 \mathrm{~b})$ and it is 


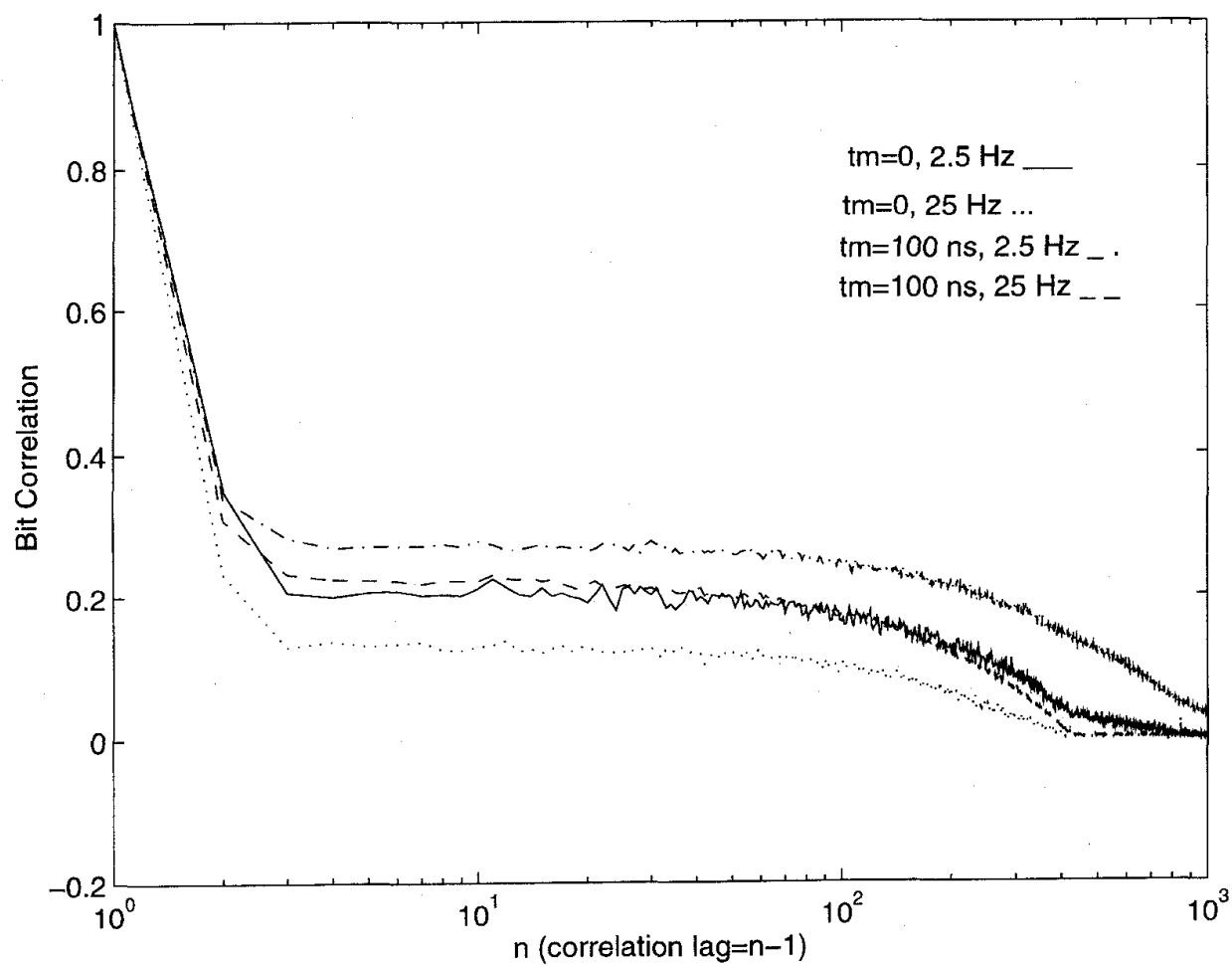

Fig. 4. Bit correlation function versus correlation lag for a $S N R \approx 30 \mathrm{~dB}$.

TABLE III

Polynomial Coefficients, in Descending Powers of $l$, FOR THE LS APPROXIMATION OF: $\log _{10}\left\{\sum_{j=l+1}^{\infty} C(j, d)\right\}$

\begin{tabular}{c|c|c|c||c|c|c}
\hline$\alpha_{3}$ & $\alpha_{2}$ & $\alpha_{1}$ & $\alpha_{0}$ & $n$ & $f_{D_{\max }}$ & $t_{m}$ \\
\hline \hline$-2.002 \mathrm{e}-02$ & $1.154 \mathrm{e}-01$ & $-4.551 \mathrm{e}-01$ & $-2.658 \mathrm{e}+00$ & 5 & $2.5 H z$ & $0 n s$ \\
\hline$-3.459 \mathrm{e}-03$ & $3.814 \mathrm{e}-02$ & $-2.332 \mathrm{e}-01$ & $-2.484 \mathrm{e}+00$ & 10 & $2.5 H z$ & $0 n s$ \\
\hline$-1.175 \mathrm{e}-03$ & $1.861 \mathrm{e}-02$ & $-1.489 \mathrm{e}-01$ & $-2.393 \mathrm{e}+00$ & 15 & $2.5 H z$ & $0 n s$ \\
\hline$-2.164 \mathrm{e}-02$ & $1.1286-01$ & $-3.706 \mathrm{e}-01$ & $-2.087 \mathrm{e}+00$ & 5 & $2.5 H z$ & $100 n s$ \\
\hline$-3.195 \mathrm{e}-03$ & $3.088 \mathrm{e}-02$ & $-1.608 \mathrm{e}-01$ & $-1.951 \mathrm{e}+00$ & 10 & $2.5 H z$ & $100 n s$ \\
\hline$-1.062 \mathrm{e}-03$ & $1.550 \mathrm{e}-02$ & $-1.060 \mathrm{e}-01$ & $-1.876 \mathrm{e}+00$ & 15 & $2.5 H z$ & $100 n s$ \\
\hline
\end{tabular}

independent of its delay spread. On the contrary, for low Doppler frequencies its memory depends on the slow fading characteristics of the channel as well as on its delay dispersion.

\section{CONCLUSION}

The computation of relevant statistics for the purpose of code performance evaluation has been obtained. The results should be seen as a guideline for the design and evaluation of efficient channel coding schemes with the aim of achieving transmission quality in implementing video and audio services, using a DECT radio system.

\section{REFERENCES}

[1] ETSI, "Digital European cordless telecommunications common interface part 2: physical layer," prETS 300, 1992.

[2] P. A. Bello, "Characterization of randomly time-variant linear channels," IEEE Trans. Commun. Syst., Dec. 1963.

[3] T. A. Wilkinson, "Channel modeling and link simulation for DECT testbed program," in Conf. Rec. Mobile Radio Personal Communications, Univ. of Warwick, U.K., Dec. 9-12, 1991, pp. 293-299.

[4] L. K. Kanal and A. R. K. Sastry, "Models for channels with memory and their applications to error control," Proc. IEEE, vol. 66, pp. 724-744, July 1978.

[5] J. P. Adoul, "Error intervals and cluster density in channel with memory," IEEE Trans. Inform. Theory, vol. IT-20, pp. 103-111, Jan. 1974.

[6] S. Tsai and P. S. Schmied, "Interleaving and error burst distribution," IEEE Trans. Commun., vol. COM-20, pp. 291-296, June 1972. 